\title{
Pharmaceutical Formulation
}

\section{Sousa e Silva JP*}

Laboratory of Pharmaceutical Technology, Faculty of Pharmacy, Department of Drug Sciences, University of Porto, Rua Jorge Viterbo Ferreira, $2284050-313$ Porto, Portugal

Pharmaceutical formulation could be simply defined as the design of dosage forms. However, this definition is too simplistic, since it does not reflect the complexity of developing pharmaceutical dosage forms. This can explain why it is easy to find references to the operation of formulation, but the definitions that describe the process are rare. Prista et al. [1], which addressed this issue, suggests that formulation could be defined as the set of operations that aim to create a physical system which contain the drug, in order to meet the quality requirements set in advance (specifications) and ensuring the maintenance of efficacy and safety characteristics of the active substance.

In the past the art of formulating had as main objective to make drug administration easier and enjoyable, but probably it always had associated, albeit incipient, the concern of stability. Currently pharmaceutical formulation, in addition to facilitate drug delivery and stability must ensure adequate bioavailability. Moreover, should enable large scale reproducibly production of medicines which comply with specification. Using preformulation as starting point, biopharmacy as support, and having as a backdrop regulatory aspects and what was defined in the project development (target product profile) pharmaceutical scientist may formulate. In practice, formulating consists in selecting the excipients, the manufacturing process, and evaluating the formulations obtained using physicochemical and pharmacotechnical tests. These tests allow selecting, optimizing and evaluating pharmaceutical preparations based on pre-established specifications. Formulation, which for many centuries was an art, begun, during the last century, to lose gradually this status in favor of a scientific -based approach, initially by controlling one factor at a time. Currently, almost all pharmaceutical technologists are aware of the disadvantages of one factor at time and recognize the advantages of statistical approach (Design of Experiments) in the formulation [2].

The concept factorial design basically consists in studying the influence of a number of factors on a response variable by varying simultaneously, but in a planned and random way (per levels), all factors. Using factorial design it is possible to evaluate the effect of each factor influencing the formulation (and possibly the interaction between factors) based on statistical analysis [3]. Factorial design may be used to select excipients and their quantities and/or conditions of the manufacturing process in preliminary or optimization studies. Partial factorial designs, which allow assessing a large number of variables by performing a relatively small number of experiments, like fractional factorial design and Plackett-Burman are used in screening studies. The Plackett-Burman design is used when only the main effects are considered significant. In this type of design (Plackett-Burman) at two levels, the number of experiments is multiple of 4 instead of being a power of 2. Full factorials are used in the optimization, where the number of variables under study tends to be lower. They have also been used artificial intelligence programs in developing formulations, such as expert systems and artificial neural networks [4]. The expert systems are computer programs that use knowledge to solve problems of inference that normally require human experts to resolve the matter. The artificial neural networks are programs that simulate biological functions of the brain such as learning.

\section{References}

1. Prista LN, Alves AC, Morgado R (1996) Formulação de Formas Farmacêuticas Sólidas. Tecnologia Farmacêutica, (4thedn), Lisboa: Fundação Calouste Gulbenkian: 2125-2150.

2. Gibson M (2001) Pharmaceutical Preformulation and Formulation: A Practical Guide from Candidate Drug Selection to Commercial Dosage Form. Informa Health Care, Leicestershire, UK.

3. Hwang R, Kowalski D (2005) Design of Experiments for Formulation Development. Pharmaceutical Technology.

4. Augsburger LL, Zellhofer MJ (2006) Encyclopedia of Pharmaceutical Technology. Informa Healthcare, UK.
*Corresponding author: Sousa e Silva JP, Laboratory of Pharmaceutical Technology, Faculty of Pharmacy, Department of Drug Sciences, University of Porto, Rua Jorge Viterbo Ferreira, 228 4050-313 Porto, Portugal, E-mail: paulo.silva@ff.up.pt

Received January 10, 2013; Accepted January 14, 2013; Published January 16 2013

Citation: Sousa e Silva JP (2013) Pharmaceutical Formulation. Pharmaceut Ana Acta 4: e145. doi:10.4172/2153-2435.1000e145

Copyright: (c) 2013 Sousa e Silva JP. This is an open-access article distributed under the terms of the Creative Commons Attribution License, which permits unrestricted use, distribution, and reproduction in any medium, provided the original author and source are credited. 